\title{
Research Paer: \\ Evaluation of the Effectiveness of Lidcomb Treatment Program on Reducing Stuttering in School-age Children
}

\section{${ }^{*}$ Elahe Farmani ${ }^{1}$}

1. Department of Speech Therapy, School of Rehabilitation Sciences, Iran University Of Medical Science, Tehran, Iran.

Citation Farmani E. Evaluation of the Effectiveness of Lidcomb Treatment Program on Reducing Stuttering in School-age Children. Jundishapur Journal of Medical Sciences. 2021; 20(2):120-127. https://doi.org/10.32598/JSMJ.20.2.3

dol' $h$ ttps://doi.org/10.32598/JSMJ.20.2.3

Received: 28 May 2020

Accepted: 28 Jun 2020

Available Online: 01 Jun 2021

Keywords:

Stuttering, School age children, Lidcomb program

\section{A B STRACT}

Background and Objectives: One of the speech disorders of school age children is stuttering. It has a great impact on communication and self-esteem in these children. There have not been many studies on the efficacy of treatment programs, especially the Lidcomb treatment program, on the severity of stuttering in school-age children. The aim of this study was to evaluate the effectiveness of the Lidcomb treatment program on stuttering in school-age children.

Subjects and Methods In this study, 15 children aged 7-11 years with stuttering were administered the Lidcomb treatment. Severity of stuttering was measured in three positions: the beginning of the study, at the end of the first phase and the end of the second phase of the Lidcomb program. Analysis of variance was performed with the SPSS software.

Results Comparison of the results showed a significant decrease in stuttering severity in school-age children following the implementation of the Lidcomb program $(P=0.000)$.

Conclusion The Lidcomb treatment program is a structured and targeted program that can be effective in reducing stuttering in school-age children.

\section{* Corresponding Author:}

Elahe Farmani, PhD.

Address: Department of Speech Therapy, School of Rehabilitation Sciences, Iran University Of Medical Science, Tehran,Iran.

Tel: +98 (21) 22227124

E-Mail: farmani.e@iums.ac.ir 


\section{مقاله بزؤهشى: \\ بررسى اثربخشى برنامه درمانى ليدكامب بر كاهش لكنت كودكان سن مدرسه

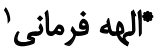

ا. كروه كفتتاردرمانى، دانشكده علوم توانبخشى، دانشكاه علوم يزشكى ايران، تهران، ايران.

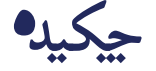

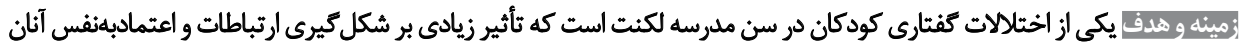

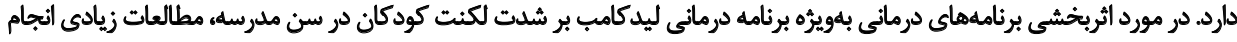

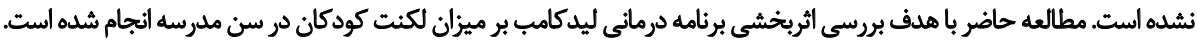

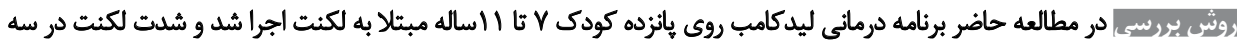

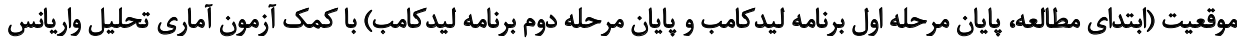

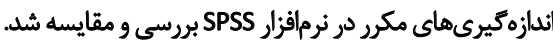

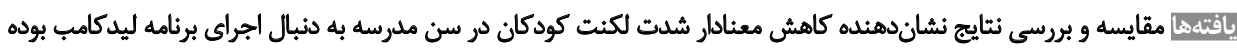

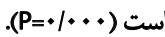

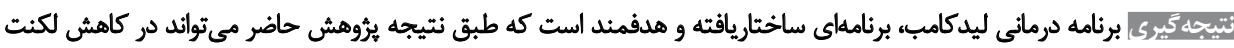
كودكان در سن مدرسه مؤثر باشد.

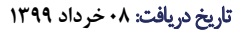

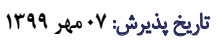

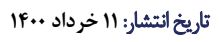

كودكان دجار لكنت در سنين ويشدبشتانى، مطالعاتى در خارج

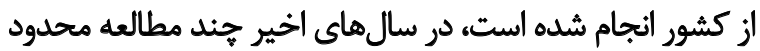

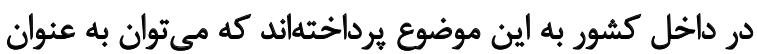

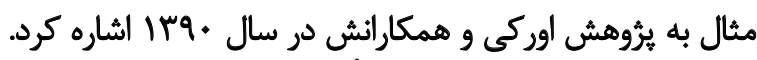

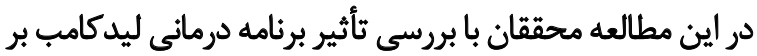

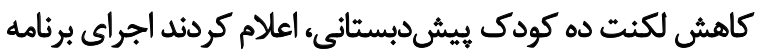

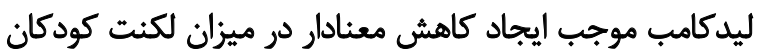

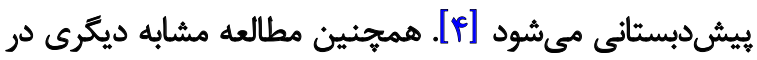

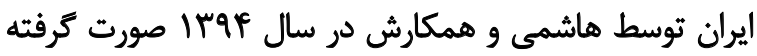

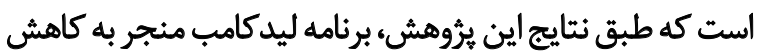

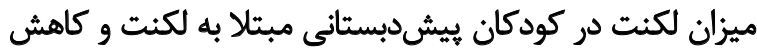

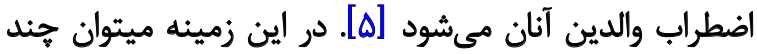

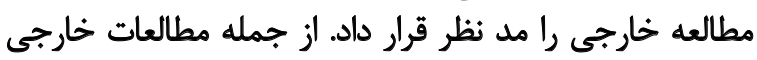

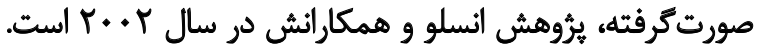

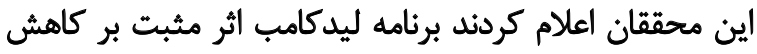

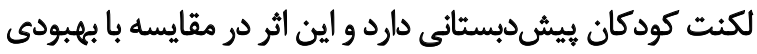

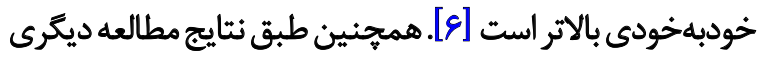

وقوع ناروانى و كسيختكى در جريان روان كفتار، لكنت نام دارد.

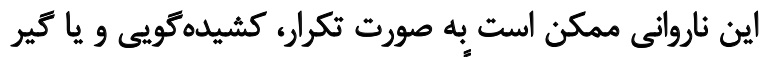

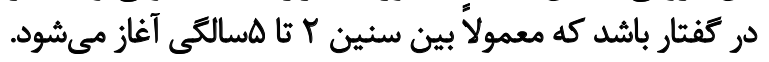

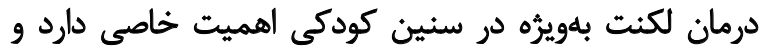

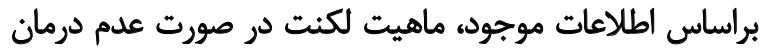

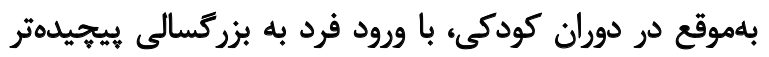

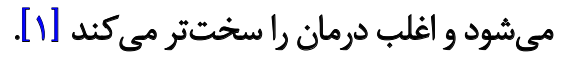
يكى از درمانهاي ارائهشده براي لكنت در سنين بيش ديشبستاني،

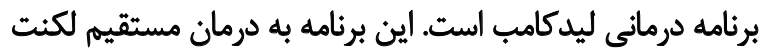

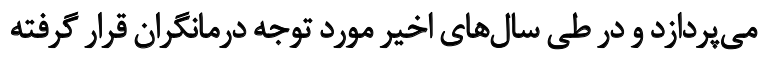

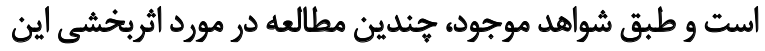

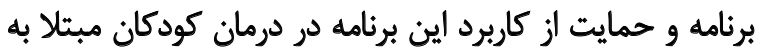

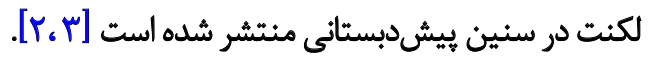
اكرجه در زمينه اثربخشى برنامه ليدكامب بر كاهش لكنت

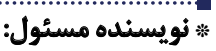

دكتر الهه فرمانى نئن

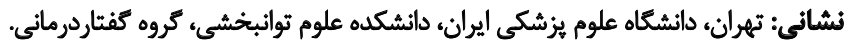

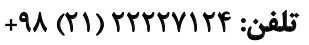
رايانامه: farmani.e@iums.ac.ir 
با تكاليف مدرسه و مسئوليتهاى يك كودك در دبستان ممكن

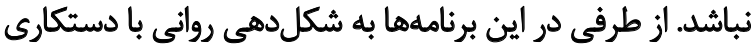

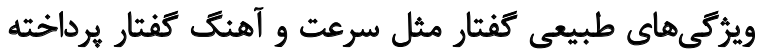

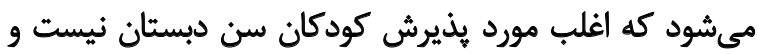

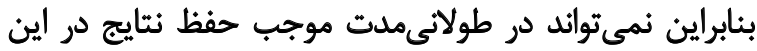

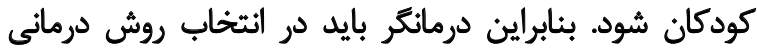

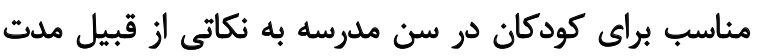

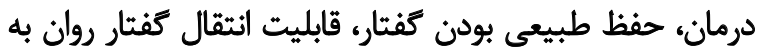
محيط خارج كلينيك و ايجاد انكيّزه در همكارى كودى وابئ و ادامه درمان توجه كند.

حال با توجه به موارد ذكرشده اين سؤال مطرح مى دئود كه

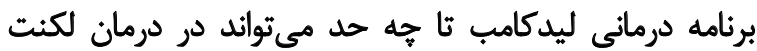

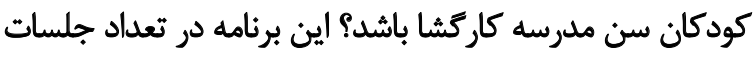

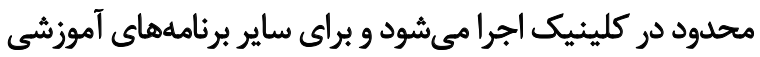

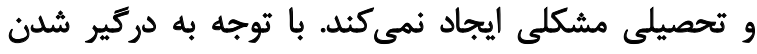

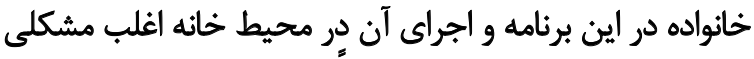

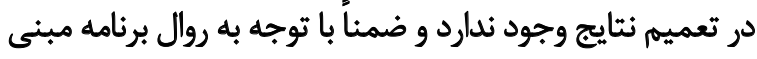

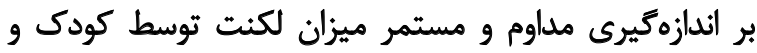

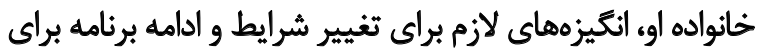

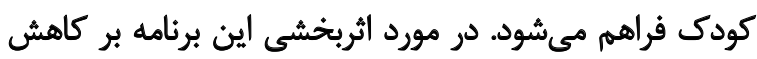

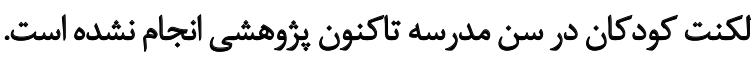

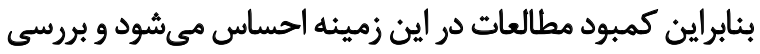

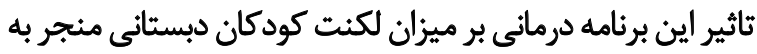

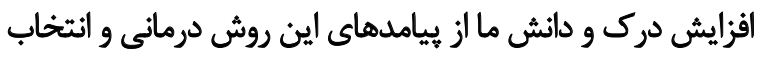

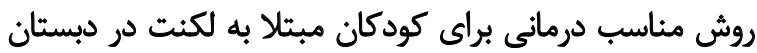

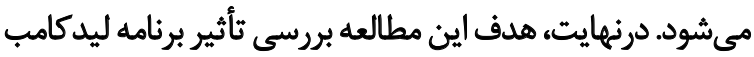
بر ميزان لكنت كودكان سن دبستان است.

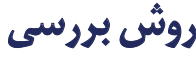

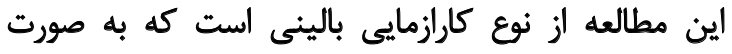

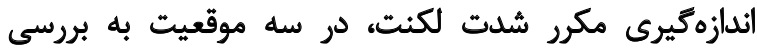

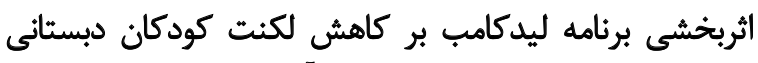

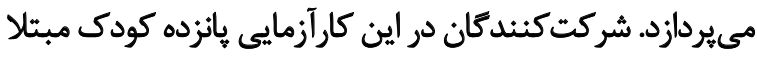

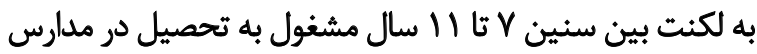

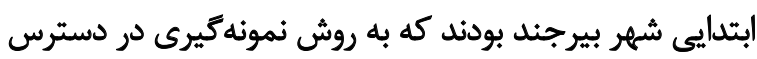

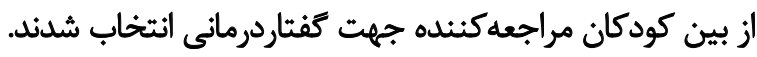

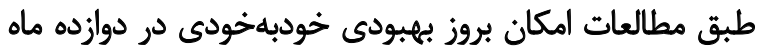

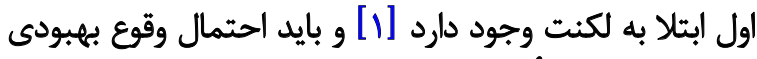

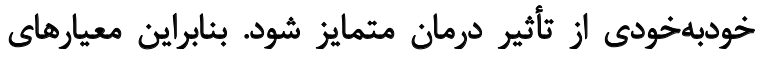

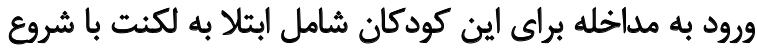

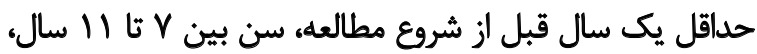

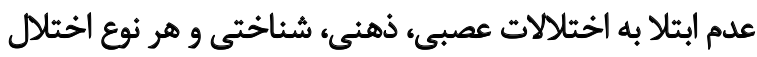
كَفتار و زبان به جز لكنت و عدم دريافت برنامه درمانى ديكر،
كه توسط جونز و همكارانش در سال ه ه •r منتشر شد، ميزان

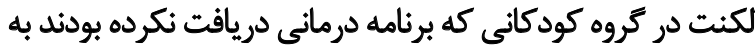

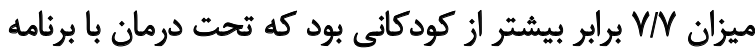

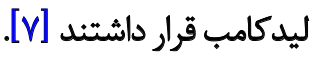

علاوه بر اين، مطالعه ديكرى نيز در اين زمينه توسط انسلو

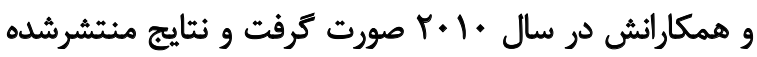

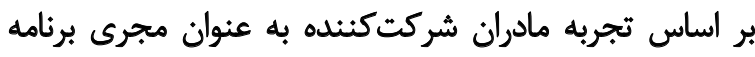

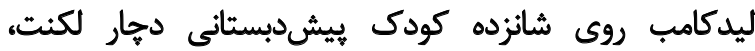

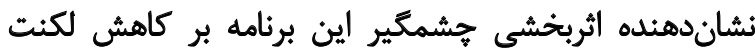

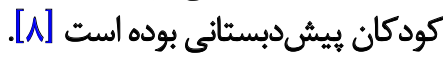

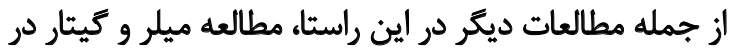

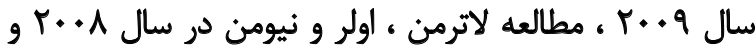

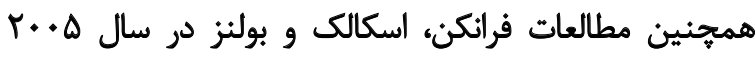

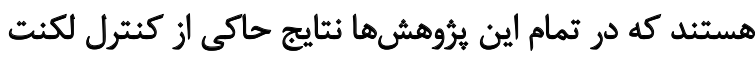

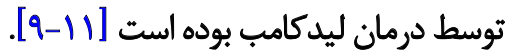
امادر اين مطالعات انجامشده اثربخشى برنامه درمانى ليدكامب

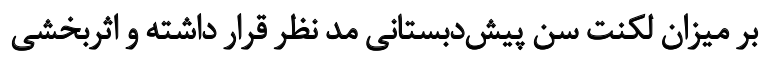

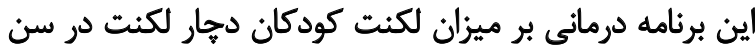

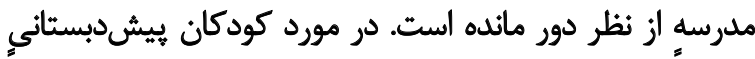

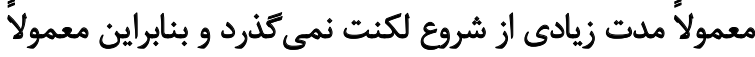

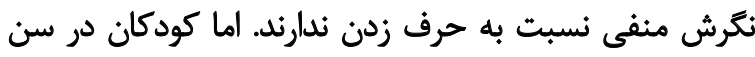

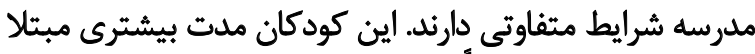

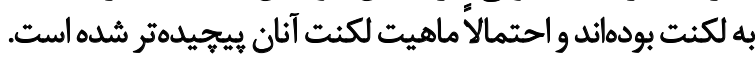

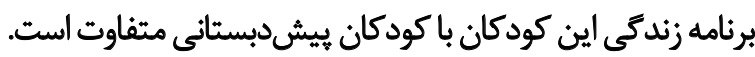

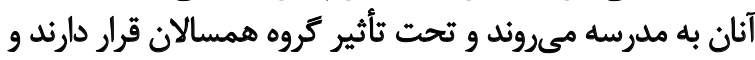

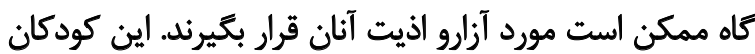

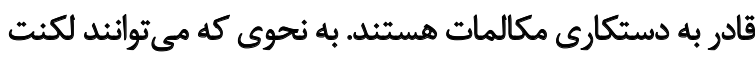

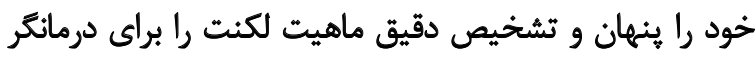

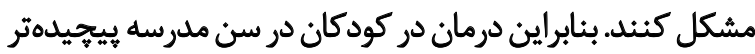

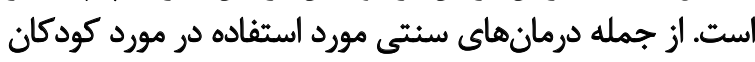

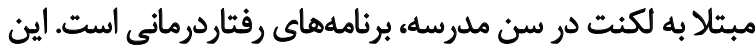

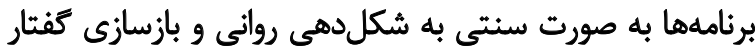

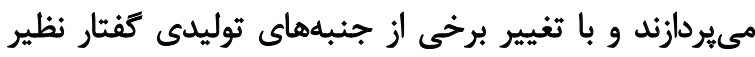

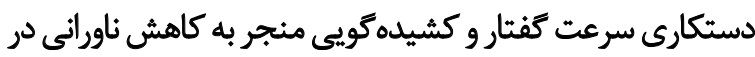

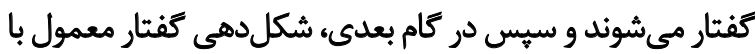

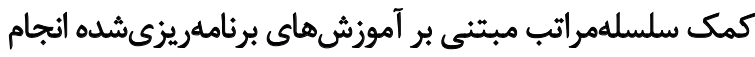

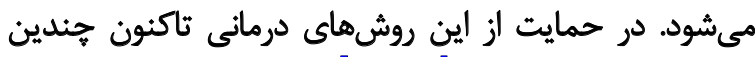

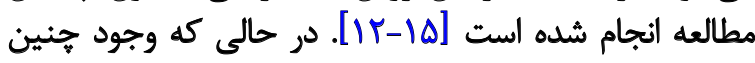

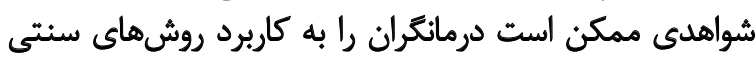

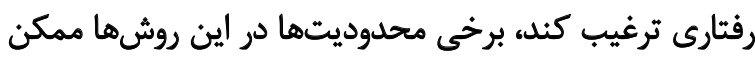

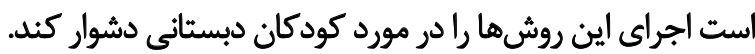

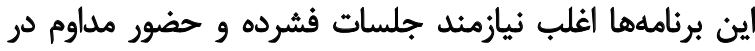

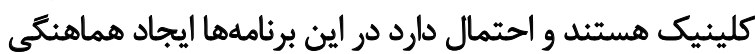


با كنترل نحوه اجراى برنامه و اقدامات صورت كرفتيه در فاصله دو

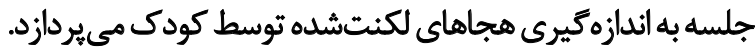

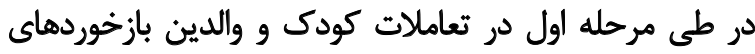

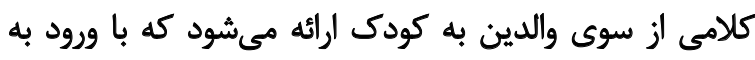

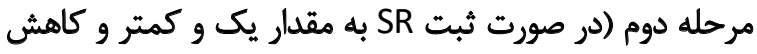

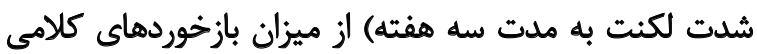

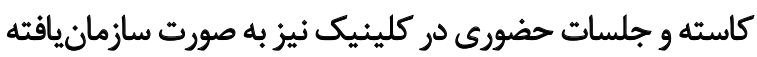

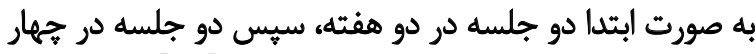

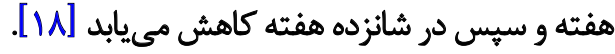
در اين برنامه جهار نوع بازخورد كلامى از سوى والدين، به كفتّار كودى تعريف شده است:

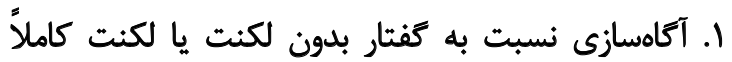

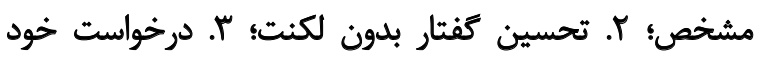

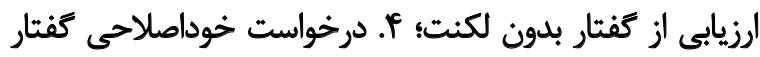

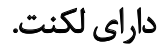

در اين مطالعه اجراى برنامه ليدكامب بدينصورت بروب بوده است:

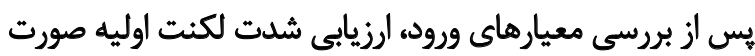

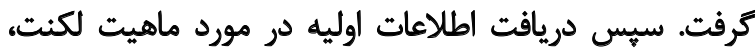

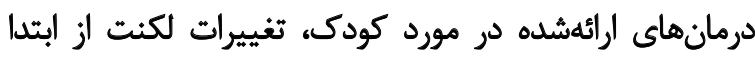

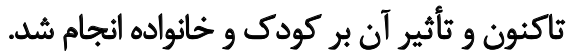

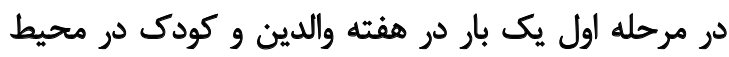

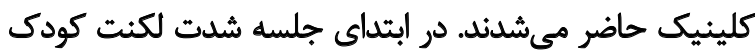

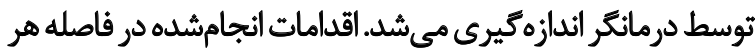

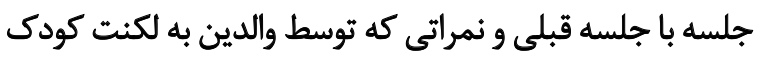

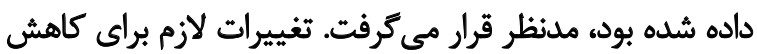

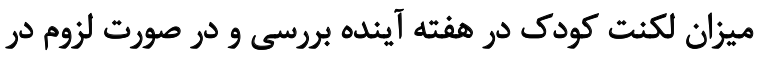

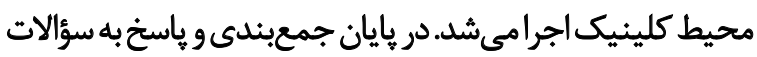

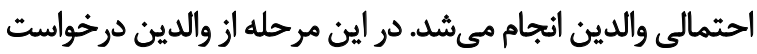

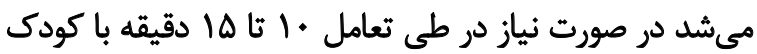

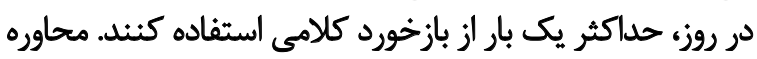

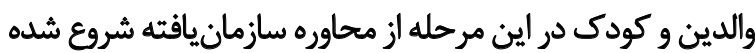

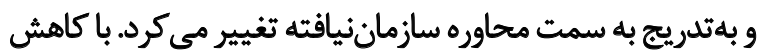

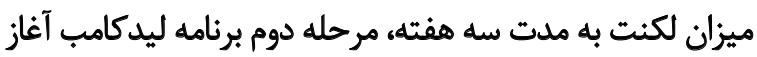

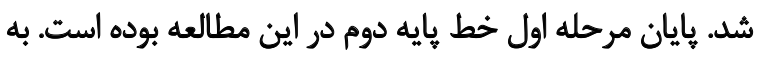

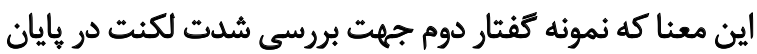

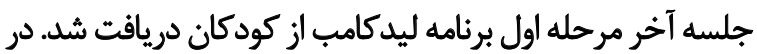

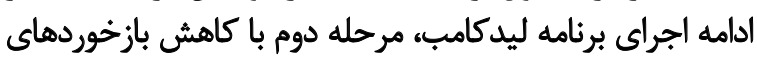

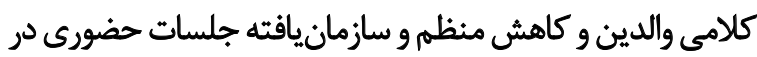

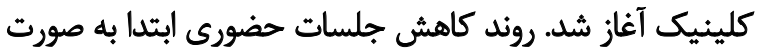

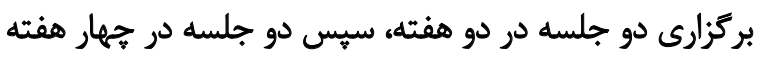

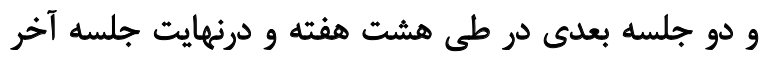
در شانزده هفته بعد بوده است. در صورتى كه در در اين جلسات
رضايت كثبى والدين جهت شركت در مداخله و نداشتن برنامه

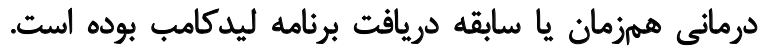

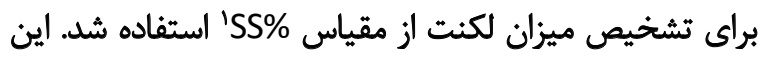

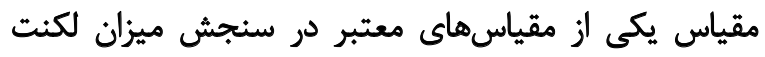

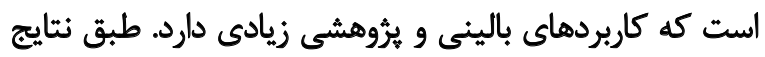

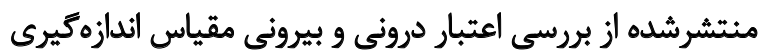

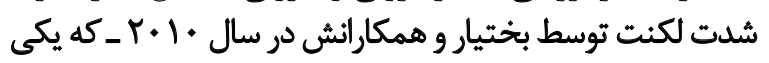

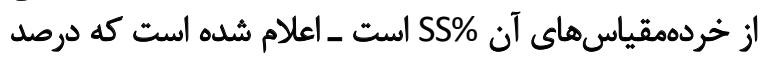

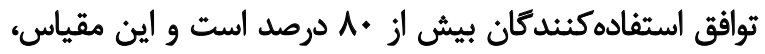

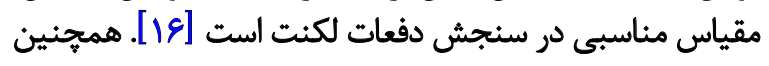

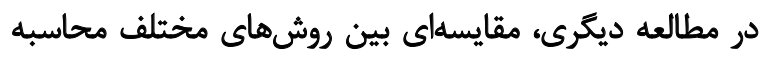

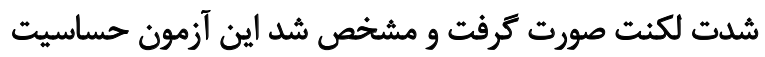

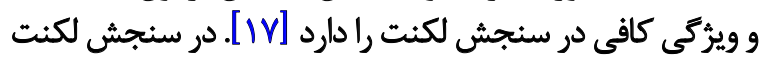

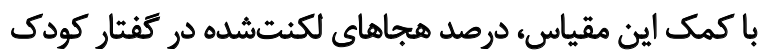

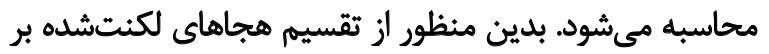

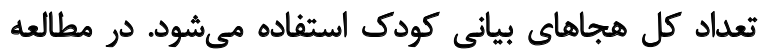

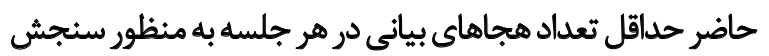

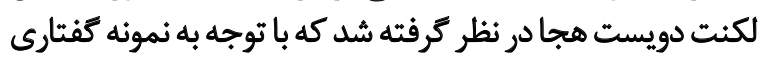

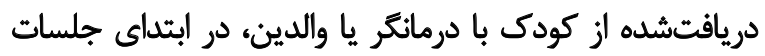

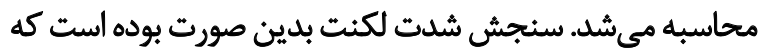

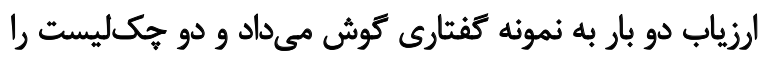

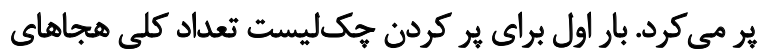

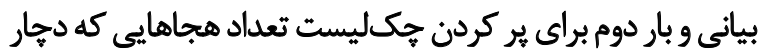

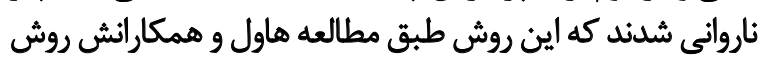

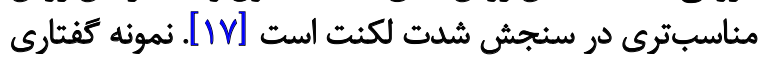

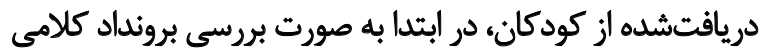

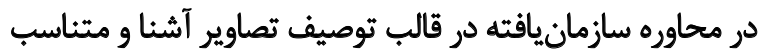

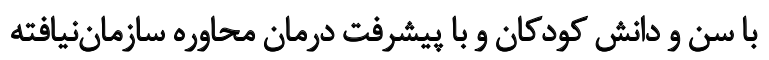

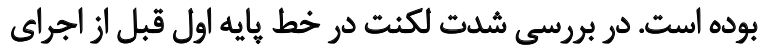

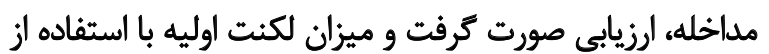

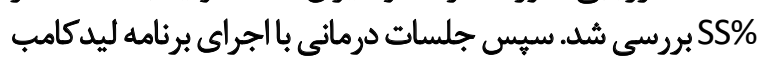

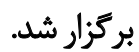

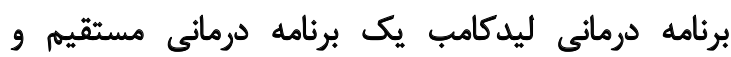

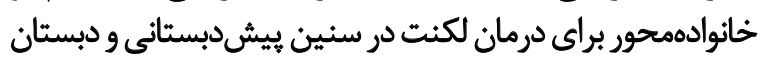

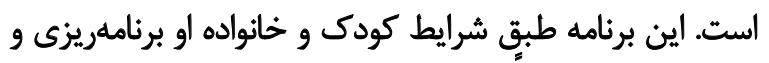

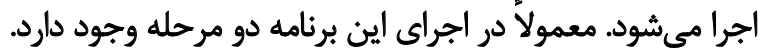

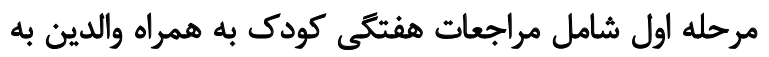

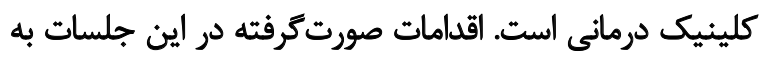

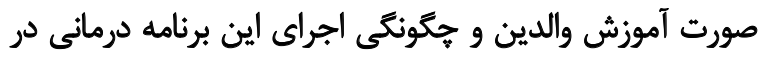

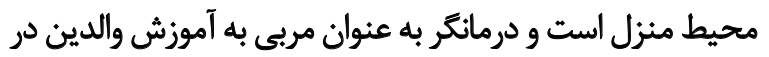

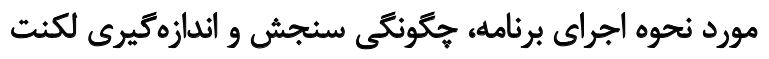

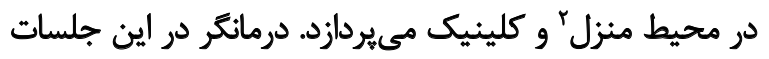


همسانى كوواريانس هاست كه در بررسى اين بيشفرض كوض از كرويت

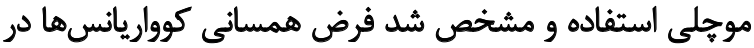

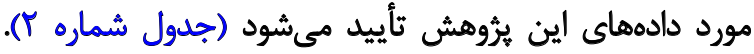

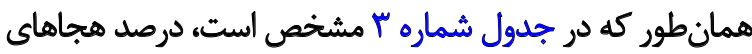

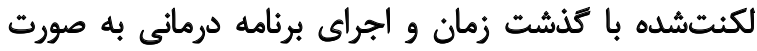

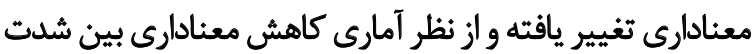

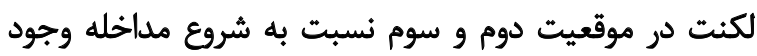

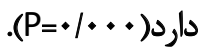

در تحليل آمارى به شيوه مذكور از آزمونهاى آمارى مختلف

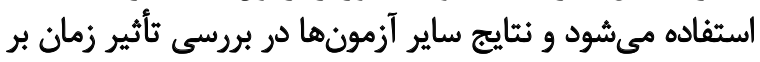

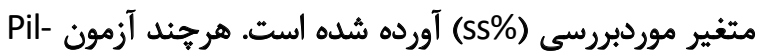

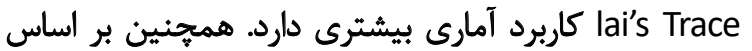

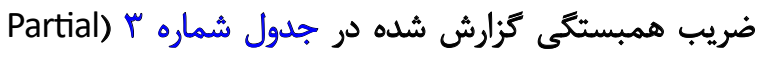
(Eta squared

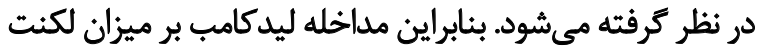

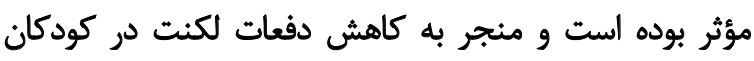

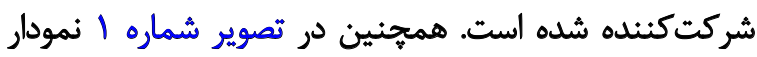
تغييرات درصد هجاهاى لكنت شده نمايش داده شده است درئ

ثج اين مطالعه با هدف بررسى اثربخشى روش درمانى ليدكامب

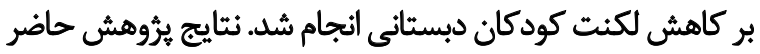

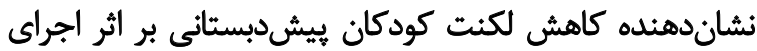

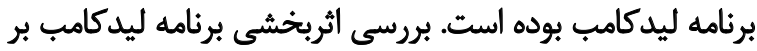

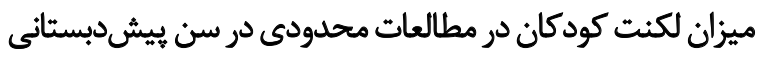

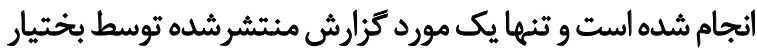

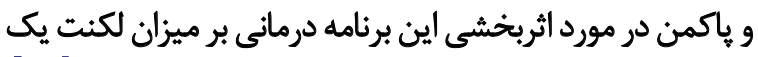

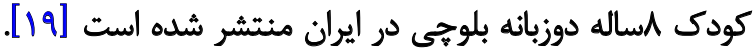

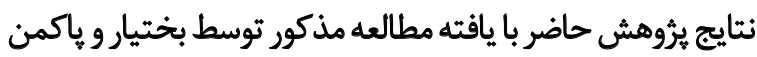

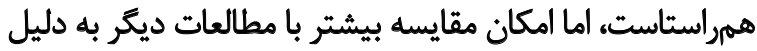

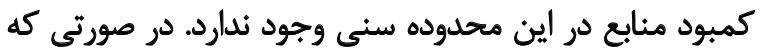

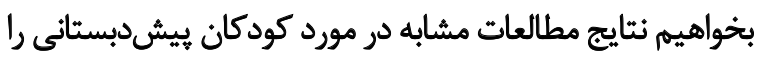

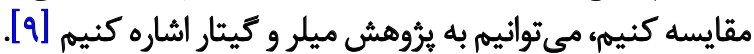

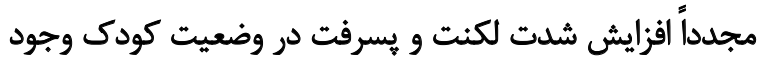

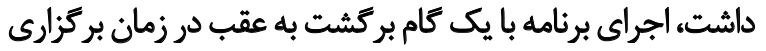

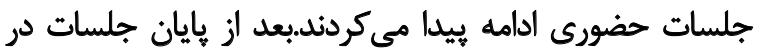

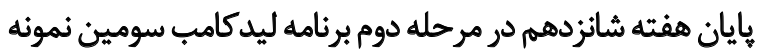

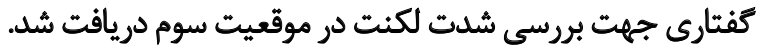

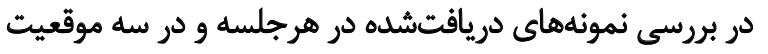

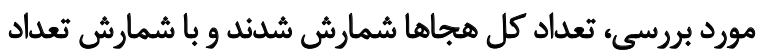
موارد لكنت و تقسيم آن بر تعداد كل هجاهاه

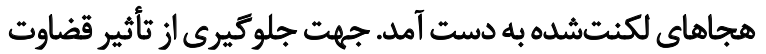

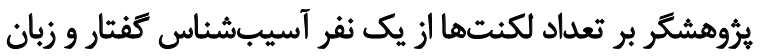

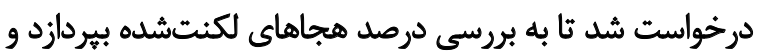

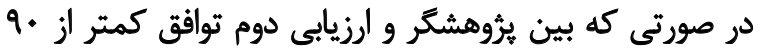

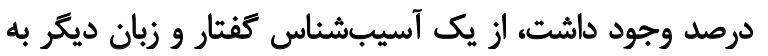

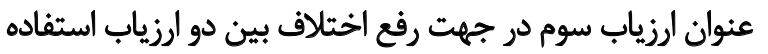

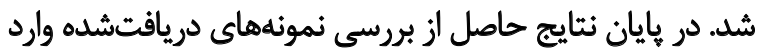

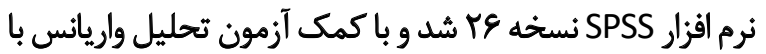
اندازميرى مكرر مورد بررسى قرار كرفت

يافتهها

طبق بررسي بهعملآمده ميانكين سنى شركت كنندكان در

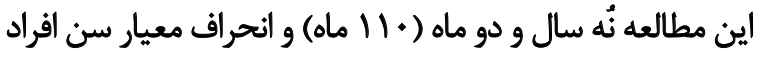

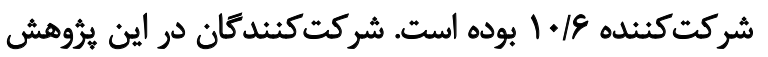

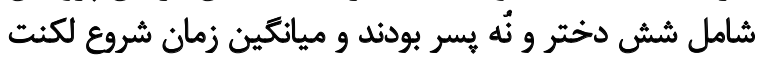

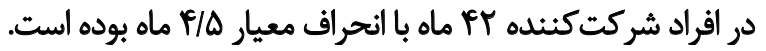

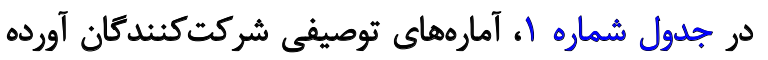

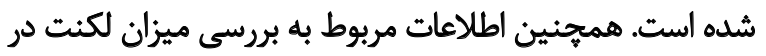

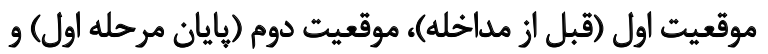

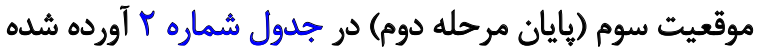

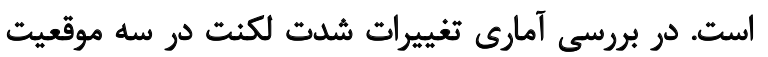

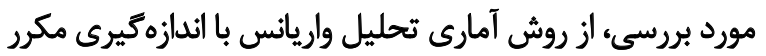

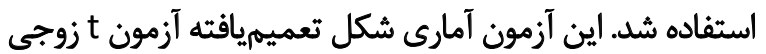

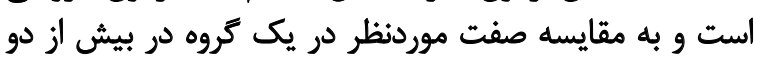

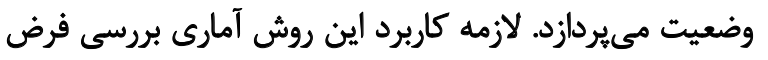

جدول (. شاخصهاى توصيفى

\begin{tabular}{|c|c|c|c|c|}
\hline ميانكين & بيشترين & كمترين & $\mathbf{N}$ & متغير \\
\hline $11 . / 94 \pm 1 . / 88$ & 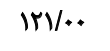 & $9 \% \%$ & 10 & سن \\
\hline$r \Delta / T+ \pm N / S$ & $p q / .$. & MI/.. & 10 & SS1 \\
\hline$r / . \pm \pm / W$ & $\Delta / .$. & $\%$ & 10 & SS2 \\
\hline \multirow[t]{2}{*}{ - RSEVI. /FOW } & $\%$ & $+\%$ & 10 & SS3 \\
\hline & & & 10 & Valid N (listwise) \\
\hline
\end{tabular}

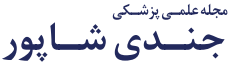




\begin{tabular}{|c|c|c|c|c|c|c|c|}
\hline & & & & & & ماجلى كرويـ & كول r. آ \\
\hline Within Subjects Effect & Mauchly's W & $\begin{array}{l}\text { Approx. Chi- } \\
\text { Square }\end{array}$ & أزاديه & معناداري & ترينهاوس- تيسر & هويين فلد & كران \\
\hline زمان & $.1+W$ & $r \cdot|A|)$ & r & .10. & $.1 \% \mathrm{~W}$ &.$/ \Delta \& A$ &.$/ 4 \cdot$. \\
\hline
\end{tabular}

جدول ب. آزمونهاى آمارى اندازهيرى مكرر

\begin{tabular}{|c|c|c|c|c|c|c|c|}
\hline & اثر & ارزش & $\mathbf{F}$ & Hypothesis df & Error df & Sig. & Partial Eta squared \\
\hline \multirow{4}{*}{ زمان } & Pillai's Trace &.$/ 9 \varphi$ & IFT/QNA & $r / \ldots$ & $\mid r / \ldots$ & $\%$ &.$/ Q 8$ \\
\hline & Wilks' Lambda & $+1 * 4+$ & $\mid r T / \Delta h \Delta$ & $r / \ldots$ & $\mid r / * \ldots$ & $+\ldots$ &.$/ 98$ \\
\hline & Hotelling's Trace & m/qm & $\mid P T / Q N A$ & $r / \%$ & $\mathrm{H} / \ldots$ & .10. &.$/ 98$. \\
\hline & Roy's Largest Root & ( & $\mid F / A M A$ & $r / \ldots$ & $\mid r / \ldots$ & $\%$ &.$/ 98$. \\
\hline
\end{tabular}

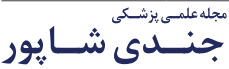

ليدكامب در يرؤوهش حاضر، به واسطه سن شركت كنيندكان نيز

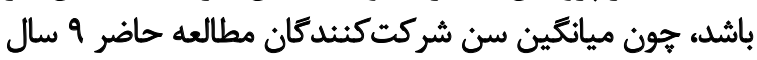

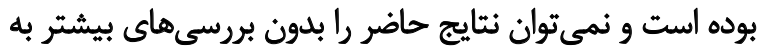

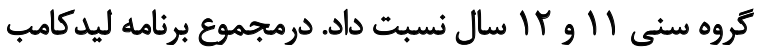

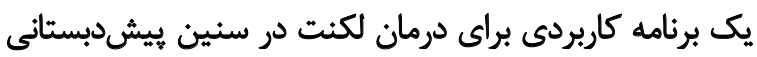

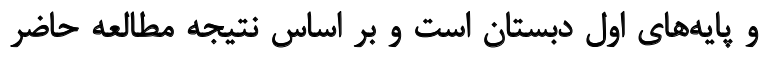

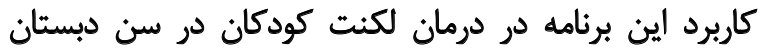

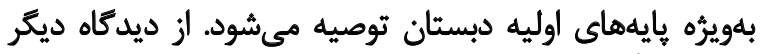

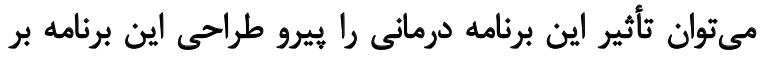

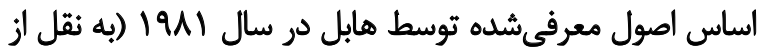

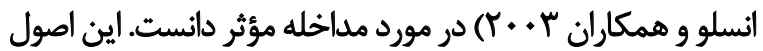

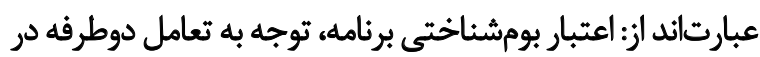

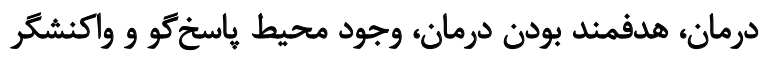

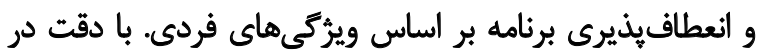

اين محققان در مطالعه خود اعلام كردند لكنت به كمك روش

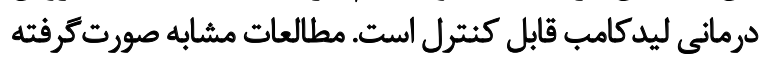

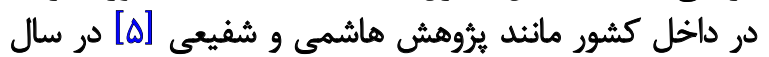

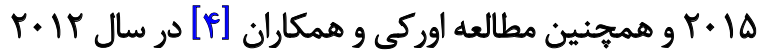

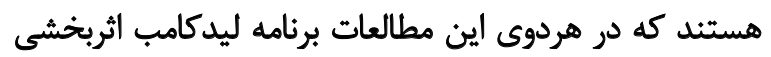

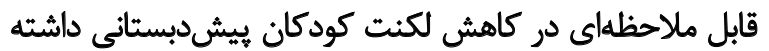

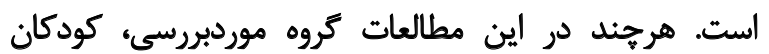

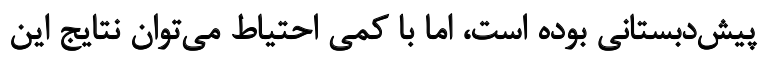

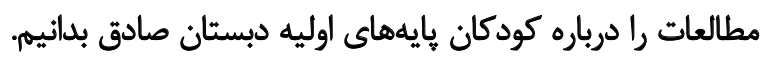

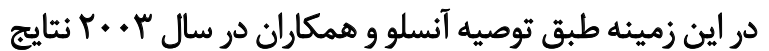

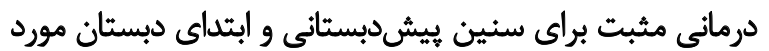

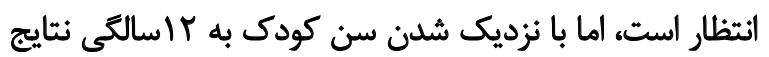

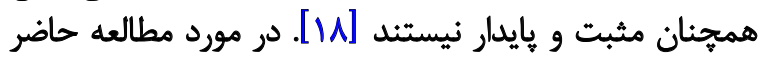

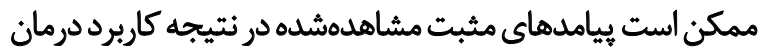

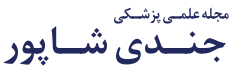

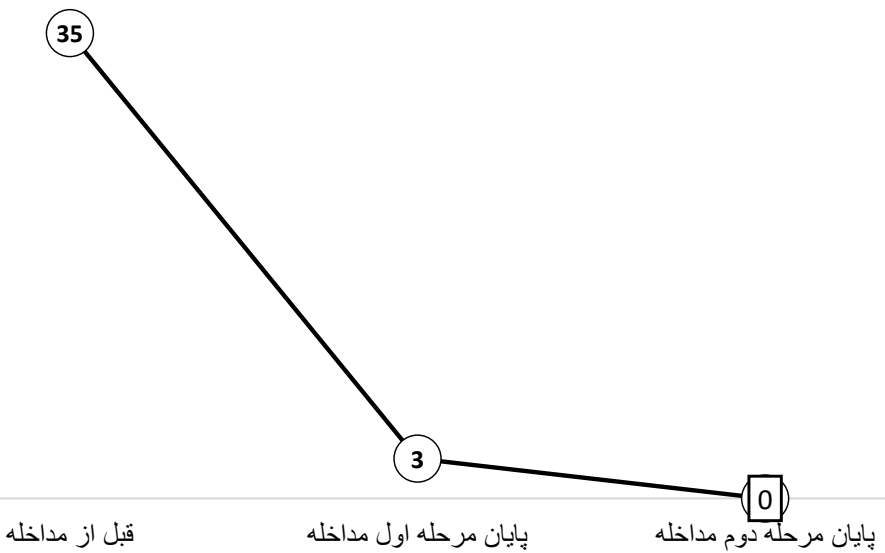




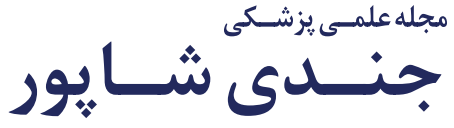

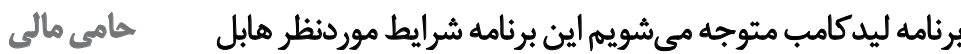

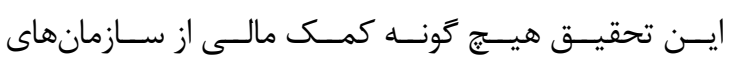

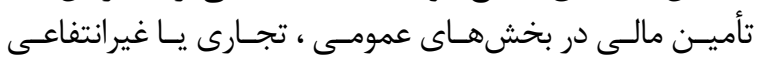

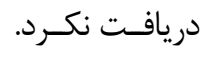

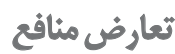

بنابر اظهار نويسندكان اين مقاله تعارض منافع ندارد.

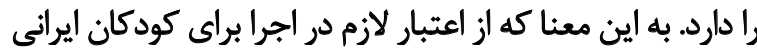

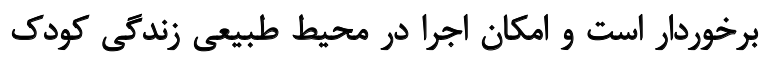

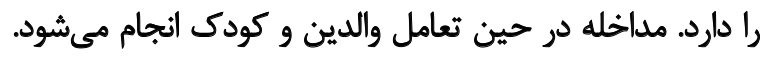

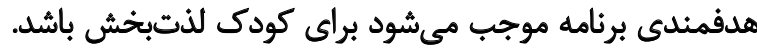

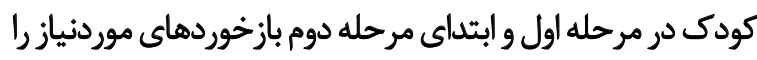

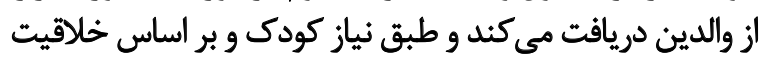

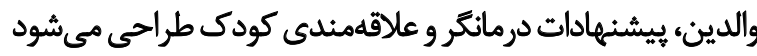

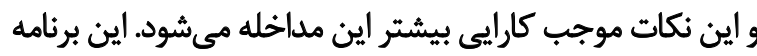

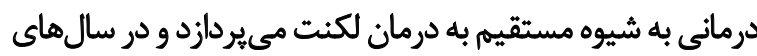

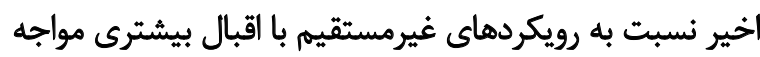

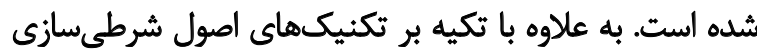

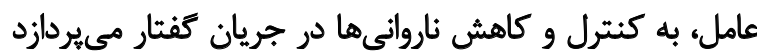

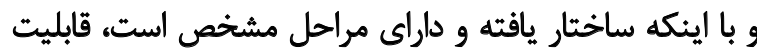

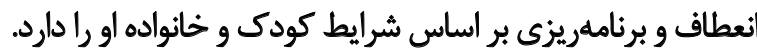

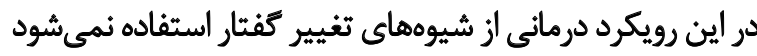

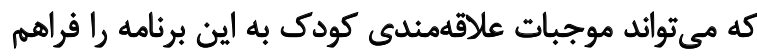

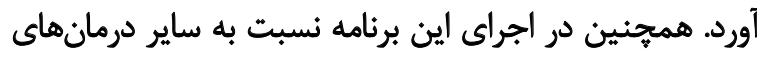

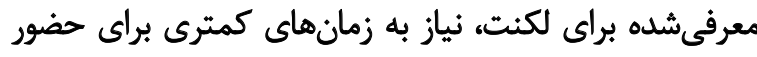

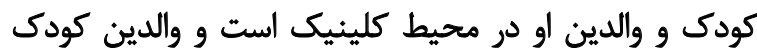

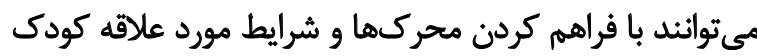

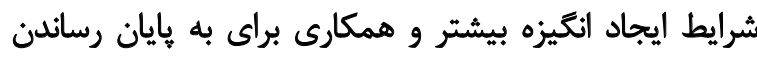

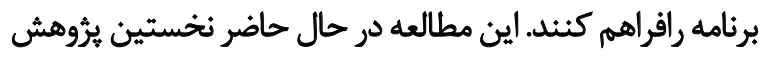

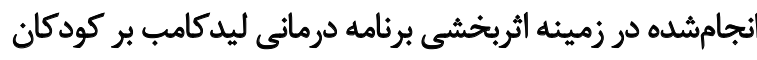
در سن دبستان است. نتيجهيَيرى

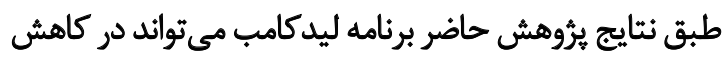

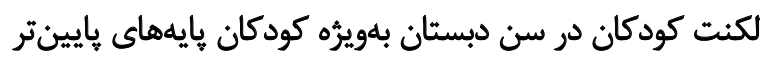

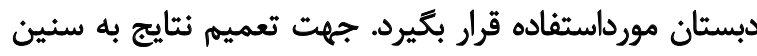

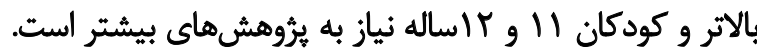

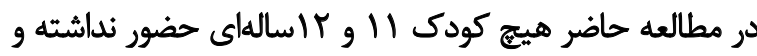

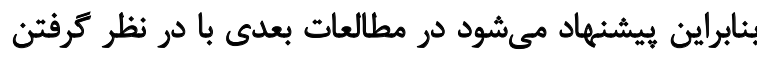

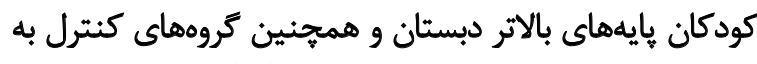

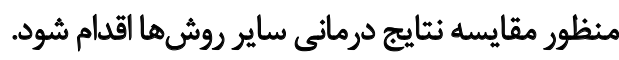

ماحظات اخلاقي - اتى

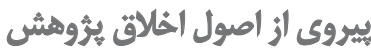

اطلاعات مربوط به ثبت اين كارآزمايى بالينى در مركز ثبت باريت كارآزمايى بالينى به شماره

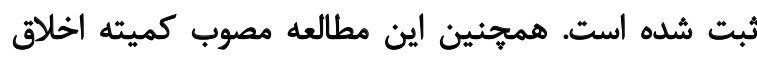

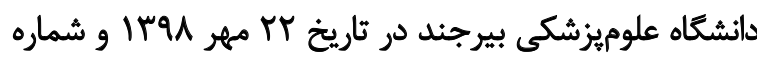




\section{Refrence}

[1] Brocklehurst PH. Stuttering prevalence, incidence and recovery rates depend on how we define it: Comment on Yairi \& Ambrose' article Epidemiology of stuttering: $21^{\text {st }}$ century advances. J Fluency Disord. 2013; 38(3):290-3. [DOI:10.1016/j.jfludis.2013.01.002] [PMID]

[2] Packman A, Onslow M, Webber M, Harrison E, Arnott S, Bridgman K, et al. The Lidcombe Program treatment guide [Internet]. 2014 [Updated 2014 January]. Available from: https://www. lidcombeprogram.org/wp-content/uploads/2015/04/LidcombeProgram-Treatment-Guide-March-2014.pdf

[3] Lattermann Ch, Shenker RC, Thordardottir E. Progression of language complexity during treatment with the Lidcombe Program for early stuttering intervention. Am J Speech Lang Pathol. 2005; 14(3):242-53. [DOI:10.1044/1058-0360(2005/024)]

[4] Oraki M, Mohseni M, Bakhtiar M. [The effectiveness of Lidcombe Program in reduction of preschool children's stuttering severity (Persian)]. J Except Child. 2013; 12(4):33-40. http://joec.ir/ article-1-171-en.html

[5] Shafiei B, Hashemi F. [Identification of efficacy of Lidcombe Program in reducing of the severity of stuttering in preschool children who stutter and their parent's anxiety reduction (Persian)]. Middle East J Disabil Stud. 2015; 5:29-38. http://jdisabilstud.org/ article-1-484-en.html

[6] Harris V, Onslow M, Packman A, Harrison E, Menzies R. An experimental investigation of the impact of the Lidcombe Program on early stuttering. J Fluency Disord. 2002; 27(3):203-14. [DOI:10.1016/S0094-730X(02)00127-4]

[7] Jones M, Onslow M, Packman A, Williams Sh, Ormond T, Schwarz I, et al. Randomized controlled trial of the Lidcombe program of early stuttering intervention. BMJ. 2005; 331(7518):659. [DOI:10.1136/bmj.38520.451840.E0] [PMID] [PMCID]

[8] Goodhue R, Onslow M, Quine S, O'Brian S, Hearne A. The Lidcombe Program of early stuttering intervention: Mothers' experiences. J Fluency Disord. 2010; 35(1):70-84. [DOI:10.1016/j. jfludis.2010.02.002] [PMID]

[9] Miller B,Guitar B. Long-term outcome of the lidcombe program for early stuttering intervention. Am J Speech Lang Pathol. 2009; 18(1):42-9. https://eric.ed.gov/?id=EJ867766

[10] Latterman Ch, Euler HA, Neuman K. A randomized control trial to investigate the impact of the Lidcombe Program on early stuttering in German-speaking preschoolers. J Fluency Disord. 2008; 33(1):52-65. [DOI:10.1016/j.jfludis.2007.12.002] [PMID]

[11] Franken MCJ, Kielstra-Van der Schalk CJ, Boelens H. Experimental treatment of early stuttering: A preliminary study. J Fluency Disord. 2005; 30(3):189-99. [DOI:10.1016/j.jfludis.2005.05.002] [PMID]

[12] Boberg K, Kully D. Long-term results of an intensive treatment program for adults and adolescents who stutter. J Speech Hear Res. 1994; 37(5):1050-9. [DOI:10.1044/jshr.3705.1050] [PMID]

[13] Hancock K, Craig A, McCready C, McCaul A, Costello D, Campbell $\mathrm{K}$, et al. Two- to six-year controlled-trial stuttering outcomes for children and adolescents. J Speech Lang Hear Res. 1998; 41(6):1242-52. [DOI:10.1044/jslhr.4106.1242] [PMID]
[14] Hancock K, Craig A. Predictors of stuttering relapse one year following treatment for children aged 9 to 14 years. J Fluency Disord. 1998; 23(1):31-48. [DOI:10.1016/S0094730X(97)00028-4]

[15] Ryan BP, Van Kirk Ryan B. Programmed stuttering treatment for children: Comparison of two establishment programs through transfer, maintenance, and follow-up. J Speech Hear Res. 1995; 38(1):61-75. [DOI:10.1044/jshr.3801.61] [PMID]

[16] Bakhtiar M, Seifpanahi S, Ansari H, Ghanadzade H, Packman A. Investigation of the reliability of the SSI-3 for preschool Persian-speaking children who stutter. J Fluency Disord. 2010; 35(2):87-91. [DOI:10.1016/j.jfludis.2010.02.003] [PMID]

[17] Howell P, Soukup-Ascencao T, Davis S, Rusbridge S. Comparison of alternative methods for obtaining severity scores of the speech of people who stutter. Clin Linguist Phon. 2011; 25(5):368-78. [DOI:10.3109/02699206.2010.538955] [PMID] [PMCID]

[18] Onslow M. Lidcombe Program of early stuttering intervention: A clinician's guide. Austin, TX: Pro-Ed; 2003. https:// books.google.com/books?id=416PpwAACAAJ\&dq

[19] Bakhtiar M, Packman A. Intervention with the Lidcombe Program for a bilingual school-age child who stutters in Iran. Folia Phoniatr Logop. 2009; 61(5):300-4. [DOI:10.1159/000241880] [PMID] 